\title{
Existence of Solutions for Fractional Boundary Value Problem with Nonlinear Derivative Dependence
}

\author{
Wenzhe Xie, ${ }^{1}$ Jing Xiao, ${ }^{2}$ and Zhiguo Luo ${ }^{1}$ \\ ${ }^{1}$ Department of Mathematics, Hunan Normal University, Changsha, Hunan 410081, China \\ ${ }^{2}$ Department of Information Engineering, Guangdong Medical College, Dongguan, Guangdong 523808, China \\ Correspondence should be addressed to Jing Xiao; xjazyh@163.com
}

Received 16 February 2014; Revised 8 April 2014; Accepted 8 April 2014; Published 27 April 2014

Academic Editor: Robert A. Van Gorder

Copyright (c) 2014 Wenzhe Xie et al. This is an open access article distributed under the Creative Commons Attribution License, which permits unrestricted use, distribution, and reproduction in any medium, provided the original work is properly cited.

We investigate the existence of solutions for fractional boundary value problem including both left and right fractional derivatives by using variational methods and iterative technique.

\section{Introduction}

Fractional differential equations appear naturally in a number of fields such as physics, chemistry, biology, economics, control theory, signal and image processing, and blood flow phenomena. During last decades, the theory of fractional differential equations is an area intensively developed, due mainly to the fact that fractional derivatives provide an excellent tool for the description of memory and hereditary properties of various materials and processes (see [1-4] and the references therein). Therein, the composition of fractional differential operators has got much attention from many scientists, mainly due to its wide applications in modeling physical phenomena exhibiting anomalous diffusion. Specifically, the models involving a fractional differential oscillator equation, which contains a composition of left and right fractional derivatives, are proposed for the description of the processes of emptying the silo [5] and the heat flow through a bulkhead filled with granular material [6], respectively. Their studies show that the proposed models based on fractional calculus are efficient and describe well the processes.

In the aspect of theory, the study of fractional boundary value problem including both left and right fractional derivatives has attracted much attention by using variational methods [7-12]. It is not easy to use the critical point theory to study the fractional differential equations including both left and right fractional derivatives, since it is often very difficult to establish a suitable space and a variational functional for the fractional boundary value problem.
For the first time, Jiao and Zhou in [7] showed that the critical point theory is an effective approach to tackle the existence of solutions for the following fractional boundary value problem:

$$
\begin{gathered}
{ }_{t} D_{T}^{\alpha}\left({ }_{0} D_{t}^{\alpha} u(t)\right)=\nabla F(t, u(t)), \quad \text { a.e. } t \in[0, T], \\
u(0)=u(T)=0,
\end{gathered}
$$

where ${ }_{0} D_{t}^{\alpha}$ and ${ }_{t} D_{T}^{\alpha}$ are the left and right Riemann-Liouville fractional derivatives of order $0<\alpha \leq 1$, respectively, $F$ : $[0, T] \times R^{N} \rightarrow R$ is a given function satisfying some assumptions, and $\nabla F(t, x)$ is the gradient of $F$ at $x$.

In [8], by performing variational methods combined with iterative technique, Sun and Zhang investigated the solvability of the following fractional boundary value problem:

$$
\begin{gathered}
\frac{d}{d x}\left(p_{0} D_{x}^{-\beta}\left(u^{\prime}(x)\right)+q_{x} D_{1}^{-\beta}\left(u^{\prime}(x)\right)\right)+f(x, u(x))=0, \\
x \in(0,1), \\
u(0)=u(1)=0,
\end{gathered}
$$

where $\beta \in(0,1), 0<p=1-q<1,{ }_{0} D_{x}^{-\beta}$, and ${ }_{x} D_{1}^{-\beta}$ denote left and right Riemann-Liouville fractional integrals of order $\beta$, respectively, and $f:[0,1] \times R \rightarrow R$ is continuous.

Motivated by the above works and $[13,14]$, in this paper, we attempt to use Mountain Pass theorem and iterative 
technique to study the existence of solutions for the following nonlinear fractional boundary value problem with dependence on fractional derivative:

$$
\begin{gathered}
{ }_{t} D_{T}^{\alpha}\left(p(t){ }_{0} D_{t}^{\alpha} u(t)\right)=f\left(t, u(t),{ }_{0} D_{t}^{\alpha} u(t)\right), \quad t \in[0, T], \\
u(0)=u(T)=0,
\end{gathered}
$$

where ${ }_{0} D_{t}^{\alpha}$ and ${ }_{t} D_{T}^{\alpha}$ are the left and right Riemann-Liouville fractional derivatives of order $1 / 2<\alpha \leq 1$, respectively, and $f \in C([0, T] \times R \times R, R)$ and $p \in C^{1}([0, T], R)$ with $p(t)>0$, for $t \in[0, T]$.

In particular, if $\alpha=1$, problem (3) reduces to the standard second order boundary value problem of the following form:

$$
\begin{gathered}
\left(p(t) u^{\prime}(t)\right)^{\prime}+f\left(t, u(t), u^{\prime}(t)\right)=0, \quad t \in[0, T] \\
u(0)=u(T)=0,
\end{gathered}
$$

where $f:[0, T] \times R \times R \rightarrow R$ is a given function.

In fact, for problem (3), due to the appearance of left and right Riemann-Liouville fractional integral, we cannot deal with problem (3) by using fixed point theorems, because it is difficult to find the equivalent integral equation corresponding to problem (3). And since problem (3) is not variational, we cannot find some functional such that its critical point is the solution corresponding to problem (3). However, when there is no presence of the fractional derivative of the solution in the nonlinearity term and $p(t)$ is a constant function, then problem (3) is studied by establishing corresponding variational structure in some suitable fractional space and applying the critical point theorems $[7,10,12]$.

In order to use variational methods, we consider a family of the following fractional boundary value problem with no dependence on the fractional derivative of the solution; that is, for each $w \in E_{0}^{\alpha}$ (which is defined in Section 2), we consider the following problem:

$$
\begin{gathered}
{ }_{t} D_{T}^{\alpha}\left(p(t){ }_{0} D_{t}^{\alpha} u(t)\right)=f\left(t, u(t),{ }_{0} D_{t}^{\alpha} w(t)\right), \quad t \in[0, T], \\
u(0)=u(T)=0 .
\end{gathered}
$$

From $[7,10,12]$, we know that problem (5) is variational and we can treat it by variational methods. Thus, for each $w \in E_{0}^{\alpha}$, we can find a solution $u_{w} \in E_{0}^{\alpha}$ with some bounds. Next, by iterative methods we can show that there exists a solution for problem (3).

\section{Preliminaries and Several Lemmas}

In this section, we introduce some basic definitions of fractional calculus and several lemmas which are used further in this paper.

Definition 1 (see [3]). Let $f$ be a function defined on $[a, b]$. The left and right Riemann-Liouville fractional integrals of order $\alpha$ for function $f$ denoted by ${ }_{a} D_{t}^{-\alpha} f(t)$ and ${ }_{t} D_{b}^{-\alpha} f(t)$, respectively, are defined by

$$
\begin{aligned}
& { }_{a} D_{t}^{-\alpha} f(t)=\frac{1}{\Gamma(\alpha)} \int_{a}^{t}(t-s)^{\alpha-1} f(s) d s, \quad t \in[a, b], \alpha>0, \\
& { }_{t} D_{b}^{-\alpha} f(t)=\frac{1}{\Gamma(\alpha)} \int_{t}^{b}(s-t)^{\alpha-1} f(s) d s, \quad t \in[a, b], \alpha>0,
\end{aligned}
$$

provided in both cases that the right-hand side is pointwise defined on $[a, b]$.

Definition 2 (see [3]). Let $f$ be a function defined on $[a, b]$. The left and right Riemann-Liouville fractional derivatives of order $\alpha$ for function $f$ denoted by ${ }_{a} D_{t}^{\alpha} f(t)$ and ${ }_{t} D_{b}^{\alpha} f(t)$, respectively, exist almost everywhere on $[a, b],{ }_{a} D_{t}^{\alpha} f(t)$, and ${ }_{t} D_{b}^{\alpha} f(t)$ and are represented by

$$
\begin{array}{r}
{ }_{a} D_{t}^{\alpha} f(t)=\frac{1}{\Gamma(n-\alpha)} \frac{d^{n}}{d t^{n}} \int_{a}^{t}(t-s)^{n-\alpha-1} f(s) d s, \\
t \in[a, b], \alpha>0, \\
{ }_{t} D_{b}^{\alpha} f(t)=\frac{(-1)^{n}}{\Gamma(n-\alpha)} \frac{d^{n}}{d t^{n}} \int_{t}^{b}(s-t)^{n-\alpha-1} f(s) d s, \\
t \in[a, b], \alpha>0 .
\end{array}
$$

Proposition 3 (see $[7,10])$. If $f \in L^{p}\left([a, b], R^{N}\right), g \in L^{q}([a, b]$, $R^{N}$ ) and $p \geq 1, q \geq 1,1 / p+1 / q \leq 1+\alpha$ or $p \neq 1, q \neq 1$, $1 / p+1 / q=1+\alpha$, then

$$
\int_{a}^{b}\left[{ }_{a} D_{t}^{-\alpha} f(t)\right] g(t) d t=\int_{a}^{b}\left[{ }_{t} D_{b}^{-\alpha} g(t)\right] f(t) d t, \quad \alpha>0 .
$$

Proposition 4 (see $[7,10])$. If $f(a)=f(b)=0, f^{\prime} \in L^{\infty}([a, b]$, $\left.R^{N}\right)$, and $g \in L^{1}\left([a, b], R^{N}\right)$, or $g(a)=g(b)=0, g^{\prime} \epsilon$ $L^{\infty}\left([a, b], R^{N}\right)$, and $f \in L^{1}\left([a, b], R^{N}\right)$, then

$$
\int_{a}^{b}\left[{ }_{a} D_{t}^{\alpha} f(t)\right] g(t) d t=\int_{a}^{b}\left[{ }_{t} D_{b}^{\alpha} g(t)\right] f(t) d t, \quad 0<\alpha \leq 1 .
$$

In order to establish a variational structure which enables us to reduce the existence of solutions of problem (5) to one of finding critical points of corresponding functional, it is necessary to construct appropriate function spaces.

Let us recall that for any fixed $t \in[0, T]$ and $1 \leq p<\infty$,

$$
\|u\|_{\infty}=\max _{t \in[0, T]}|u(t)|, \quad\|u\|_{L^{p}}=\left(\int_{0}^{T}|u(s)|^{p} d s\right)^{1 / p} .
$$

Definition 5. Let $0<\alpha \leq 1$. The fractional derivative space $E_{0}^{\alpha}$ is defined by the closure of $C_{0}^{\infty}([0, T], R)$ with respect to the weighted norm

$$
\|u\|_{\alpha}=\left(\int_{0}^{T} p(t)\left|{ }_{0} D_{t}^{\alpha} u(t)\right|^{2} d t+\int_{0}^{T}|u(t)|^{2} d t\right)^{1 / 2}
$$

$$
\forall u \in E_{0}^{\alpha} \text {, }
$$


where denote by $C_{0}^{\infty}([0, T], R)$ the set of all functions $u \in$ $C^{\infty}([0, T], R)$ with $u(0)=u(T)=0$.

Lemma 6 (see [7]). Let $1 / 2<\alpha \leq 1$; for all $u \in E_{0}^{\alpha}$, one has

(i)

$$
\|u\|_{L^{2}} \leq \frac{T^{\alpha}}{\Gamma(\alpha+1)}\left\|_{0} D_{t}^{\alpha} u(t)\right\|_{L^{2}}
$$

(ii)

$$
\|u\|_{\infty} \leq \frac{T^{\alpha-1 / 2}}{\Gamma(\alpha) \sqrt{2 \alpha-1}}\left\|_{0} D_{t}^{\alpha} u(t)\right\|_{L^{2}} .
$$

Remark 7. Let $m:=\min _{t \in[0, T]} p(t)$, by (12) and (13); then

$$
\begin{aligned}
\|u\|_{L^{2}} & \leq \frac{T^{\alpha}}{\Gamma(\alpha+1) \sqrt{m}}\left(\int_{0}^{T} p(t)\left|{ }_{0} D_{t}^{\alpha} u(t)\right|^{2} d t\right)^{1 / 2}, \\
\|u\|_{\infty} & \leq \frac{T^{\alpha-1 / 2}}{\Gamma(\alpha) \sqrt{m(2 \alpha-1)}}\left(\int_{0}^{T} p(t)\left|{ }_{0} D_{t}^{\alpha} u(t)\right|^{2} d t\right)^{1 / 2} .
\end{aligned}
$$

At this point, by (14), we can consider $E_{0}^{\alpha}$ with respect to the norm

$$
\|u\|_{\alpha}=\left(\int_{0}^{T} p(t)\left|{ }_{0} D_{t}^{\alpha} u(t)\right|^{2} d t\right)^{1 / 2}, \quad \forall u \in E_{0}^{\alpha},
$$

which is equivalent to (11).

Similarly to some results in [7], the properties of the fractional derivative space $E_{0}^{\alpha}$ are given, as follows.

Lemma 8. Let $0<\alpha \leq 1$. The fractional derivative space $E_{0}^{\alpha}$ is a reflexive and a separable Banach space.

Lemma 9. Assume that $1 / 2<\alpha \leq 1$ and the sequence $\left\{u_{k}\right\}$ converges weakly to $u$ in $E_{0}^{\alpha}$; that is, $u_{k} \rightarrow u$. Then $u_{k} \rightarrow u$ in $C\left([0, T], R^{N}\right)$; that is, $\left\|u-u_{k}\right\|_{\infty} \rightarrow 0$, as $k \rightarrow \infty$.

One is now in a position to give the definition for the solution of problem (3).

Definition 10. A function $u \in E_{0}^{\alpha}$ is called a solution of problem (3), if

(i) ${ }_{t} D_{T}^{\alpha-1}\left(p(t){ }_{0} D_{t}^{\alpha} u(t)\right)$ and ${ }_{0} D_{t}^{\alpha-1} u(t)$ are derivatives
for all $t \in[0, T]$,

(ii) $u$ satisfies (3).

Definition 11. A function $u \in E_{0}^{\alpha}$ is called a weak solution of problem (3), if

$$
\begin{aligned}
& \int_{0}^{T} p(t){ }_{0} D_{t}^{\alpha} u(t){ }_{0} D_{t}^{\alpha} v(t) d t \\
& \quad-\int_{0}^{T} f\left(t, u(t),{ }_{0} D_{t}^{\alpha} u(t)\right) v(t) d t=0,
\end{aligned}
$$

for all $v(t) \in E_{0}^{\alpha}$.
Associated to the boundary value problem (5), for given $w(t) \in E_{0}^{\alpha}$, we have the functional $I_{w}(u): E_{0}^{\alpha} \rightarrow R$ defined by

$$
\begin{aligned}
I_{w}(u)= & \frac{1}{2} \int_{0}^{T} p(t)\left|{ }_{0} D_{t}^{\alpha} u(t)\right|^{2} d t \\
& -\int_{0}^{T} F\left(t, u(t),{ }_{0} D_{t}^{\alpha} w(t)\right) d t,
\end{aligned}
$$

where $F(t, \xi, \zeta)=\int_{0}^{\xi} f(t, s, \zeta) d s$ and $f \in C([0, T] \times R \times R, R)$. Clearly, by the continuity hypothesis on $f$ and $p$, we have $I_{w} \in$ $C^{1}\left(E_{0}^{\alpha}, R\right)$ and for all $v(t) \in E_{0}^{\alpha}$,

$$
\begin{aligned}
I_{w}^{\prime}(u) v= & \int_{0}^{T} p(t){ }_{0} D_{t}^{\alpha} u(t){ }_{0} D_{t}^{\alpha} v(t) d t \\
& -\int_{0}^{T} f\left(t, u(t),{ }_{0} D_{t}^{\alpha} w(t)\right) v(t) d t .
\end{aligned}
$$

Moreover, similarly to the proof of [7, Theorem 5.1], we know that the critical point of $I_{w}$ is a solution of problem (5).

\section{Main Results}

First, we make the following assumptions:

$\left(H_{1}\right) f(t, \xi, \zeta)=g(t, \xi, \zeta)+h(t) \zeta$, where $g(t, \xi, \zeta)=o(|\xi|)$ as $\xi \rightarrow 0$ uniformly for $t \in[0, T], \zeta \in R$, and $h(t) \in$ $C[0, T]$; denote $C_{h}=\max _{t \in[0, T]}|h(t)|$.

$\left(H_{2}\right)$ There exist constants $C_{0}>0$ and $r>1$ such that

$$
|g(t, \xi, \zeta)| \leq C_{0}\left(1+|\xi|^{r}\right), \quad \forall t \in[0, T], \xi \in R, \zeta \in R .
$$

$\left(H_{3}\right)$ There exist constants $\mu>2$ and $\xi_{0}>0$ such that

$$
\mu G(t, \xi, \zeta) \leq \xi g(t, \xi, \zeta), \quad \forall t \in[0, T],|\xi| \geq \xi_{0}, \zeta \in R .
$$

$\left(H_{4}\right)$ There exist positive constants $C_{1}, C_{2}>0$ such that

$$
G(t, \xi, \zeta) \geq C_{1}|\xi|^{\mu}-C_{2}, \quad \forall t \in[0, T], \xi \in R, \zeta \in R,
$$

where $G(t, \xi, \zeta)=\int_{0}^{\xi} g(t, s, \zeta) d s$ and $g \in C([0, T] \times$ $R \times R, R)$.

$\left(H_{5}\right)$ For $t \in[0, T], \xi_{1}, \xi_{2} \in\left[-\rho_{1}, \rho_{1}\right], \zeta_{1}, \zeta_{2} \in R$, the function $g$ satisfies the following Lipschitz conditions:

$$
\left|g\left(t, \xi_{1}, \zeta_{1}\right)-g\left(t, \xi_{2}, \zeta_{2}\right)\right| \leq L_{1}\left|\xi_{1}-\xi_{2}\right|+L_{2}\left|\zeta_{1}-\zeta_{2}\right|,
$$

where $L_{1}, L_{2}>0$ and $\rho_{1}$ is a positive constant, which is given in (49).

We note that it obviously follows from $\left(H_{1}\right)$ and $\left(H_{2}\right)$ that for all $\varepsilon>0$, there exists a positive constant $C_{\varepsilon}$, independent of $w$, such that

$$
|g(t, \xi, \zeta)| \leq \varepsilon|\xi|+C_{\varepsilon}|\xi|^{r} .
$$

The main result of this paper is the following. 
Theorem 12. Assume that $\left(H_{1}\right)-\left(H_{5}\right)$ hold and that $1 / 2-$ $1 / \mu>C_{h} T^{\alpha} / m \Gamma(\alpha+1)$. If there exist $\bar{b} \in(0,1 / 2)$ and $\varepsilon \in$ $\left(0,(1-2 \bar{b}) m[\Gamma(\alpha+1)]^{2} / T^{2 \alpha}\right)$ such that

$$
\begin{gathered}
{\left[\frac{1}{2}-\bar{b}-\frac{\varepsilon T^{2 \alpha}}{2 m[\Gamma(\alpha+1)]^{2}}\right] \frac{(r+1)[\Gamma(\alpha) \sqrt{m(2 \alpha-1)}]^{r+1}}{C_{\varepsilon} T^{(\alpha-1 / 2)(r+1)+1}}} \\
>\left[\frac{C_{h} T^{\alpha} R}{\bar{b} m \Gamma(\alpha+1)}\right]^{r-1} \\
L:=\frac{T^{\alpha}\left(L_{2}+C_{h}\right) \Gamma(\alpha+1)}{m[\Gamma(\alpha+1)]^{2}-L_{1} T^{2 \alpha}} \in(0,1)
\end{gathered}
$$

then problem (3) has one nontrivial solution.

Proof. In order to prove Theorem 12, we proceed by three steps.

Step 1. Let $w \in E_{0}^{\alpha}$; with $\|w\|_{\alpha} \leq K$, which is given in (48), we show that $I_{w}$ has a nontrivial critical point in $E_{0}^{\alpha}$ by the Mountain Pass theorem.

Firstly, by $\left(H_{1}\right)$ and (24) we have

$$
\begin{gathered}
|f(t, \xi, \zeta)| \leq \varepsilon|\xi|+C_{\varepsilon}|\xi|^{r}+C_{h}|\zeta| \\
|F(t, \xi, \zeta)| \leq \frac{\varepsilon}{2}|\xi|^{2}+\frac{C_{\varepsilon}}{r+1}|\xi|^{r+1}+C_{h}|\zeta||\xi| .
\end{gathered}
$$

By Remarks (15), (18), (28), and Hölder inequality

$$
\begin{aligned}
I_{w}(u) \geq & \left.\left.\frac{1}{2} \int_{0}^{T} p(t)\right|_{0} D_{t}^{\alpha} u(t)\right|^{2} d t \\
& -\frac{\varepsilon}{2} \int_{0}^{T}|u(t)|^{2} d t-\frac{C_{\varepsilon}}{r+1} \int_{0}^{T}|u(t)|^{r+1} d t \\
& -C_{h} \int_{0}^{T}\left|{ }_{0} D_{t}^{\alpha} w(t)\right| \cdot|u(t)| d t \\
\geq & \frac{1}{2} \int_{0}^{T} p(t)\left|{ }_{0} D_{t}^{\alpha} u(t)\right|^{2} d t \\
& -\left.\left.\frac{\varepsilon T^{2 \alpha}}{2 m[\Gamma(\alpha+1)]^{2}} \int_{0}^{T} p(t)\right|_{0} D_{t}^{\alpha} u(t)\right|^{2} d t \\
& -\frac{C_{\varepsilon} T^{(\alpha-1 / 2)(r+1)+1}}{(r+1)[\Gamma(\alpha) \sqrt{m(2 \alpha-1)}]^{r+1}} \\
& \times\left(\left.\left.\int_{0}^{T} p(t)\right|_{0} D_{t}^{\alpha} u\right|^{2} d t\right) \\
& -\frac{C_{h} T^{\alpha}}{m \Gamma(\alpha+1) / 2}\|w\|_{\alpha} \cdot\|u\|_{\alpha}
\end{aligned}
$$

$$
\begin{aligned}
& \geq\left[\frac{1}{2}-\bar{b}-\frac{\varepsilon T^{2 \alpha}}{2 m[\Gamma(\alpha+1)]^{2}}\right. \\
& \left.\quad-\frac{C_{\varepsilon} T^{(\alpha-1 / 2)(r+1)+1}}{(r+1)[\Gamma(\alpha) \sqrt{m(2 \alpha-1)}]^{r+1}}\|u\|_{\alpha}^{r-1}\right]\|u\|_{\alpha}^{2} \\
& \quad+\left[\bar{b}\|u\|_{\alpha}-\frac{C_{h} T^{\alpha} K}{m \Gamma(\alpha+1)}\right]\|u\|_{\alpha} .
\end{aligned}
$$

By (25) we can choose $\rho>0$ such that

$$
\begin{aligned}
\frac{1}{2}-\bar{b}-\frac{\varepsilon T^{2 \alpha}}{2 m[\Gamma(\alpha+1)]^{2}}>\frac{C_{\varepsilon} T^{(\alpha-1 / 2)(r+1)+1}}{(r+1)[\Gamma(\alpha) \sqrt{m(2 \alpha-1)}]^{r+1}} \rho^{r-1}, \\
\rho>\frac{C_{h} T^{\alpha} K}{\bar{b} m \Gamma(\alpha+1)} .
\end{aligned}
$$

Hence, let $u \in E_{0}^{\alpha}$; with $\|u\|_{\alpha}=\rho$, we know that there exist $\alpha_{1}>0$, such that for $\|u\|_{\alpha}=\rho, I_{w}(u) \geq \alpha_{1}$ uniformly for $w \epsilon$ $E_{0}^{\alpha}$.

Secondly, for given $u^{*} \in E_{0}^{\alpha}$, with $\left\|u^{*}\right\|_{\alpha}=1$, it follows from $\left(H_{1}\right)$ and $\left(H_{4}\right)$ that

$$
F(t, \xi, \zeta) \geq C_{1}|\xi|^{\mu}+h(t) \zeta \xi-C_{2} .
$$

Then we have that for $\tau>0$

$$
\begin{aligned}
I_{w}\left(\tau u^{*}\right)= & \frac{\tau^{2}}{2}\left\|u^{*}\right\|_{\alpha}^{2}-\int_{0}^{T} F\left(t, \tau u^{*},{ }_{0} D_{t}^{\alpha} w(t)\right) d t \\
\leq & \frac{\tau^{2}}{2}-C_{1} \tau^{\mu} \int_{0}^{T}\left|u^{*}\right|^{\mu} d t \\
& +\tau C_{h} \int_{0}^{T}\left|D_{0}^{\alpha} D_{t}^{\alpha} w\right| \cdot\left|u^{*}\right| d t+C_{2} T \\
\leq & \frac{\tau^{2}}{2}-C_{1} \tau^{\mu} \int_{0}^{T}\left|u^{*}\right|^{\mu} d t+\tau C_{h} \frac{T^{\alpha} K}{m \Gamma(\alpha+1)}+C_{2} T .
\end{aligned}
$$

Since $\mu>2$, taking $\tau$ large enough and letting $e=\tau u^{*}$, then $I_{w}(e)<0$ with $\|e\|_{\alpha}>\rho$.

Thirdly, we show that $I_{w}$ satisfies the Palais-Smale condition.

Let $\left\{u_{k}\right\} \subset E_{0}^{\alpha}$, such that

$$
\left|I_{w}\left(u_{k}\right)\right| \leq \bar{K}, \quad \lim _{k \rightarrow \infty} I_{w}^{\prime}\left(u_{k}\right)=0, \quad \text { for some } \bar{K}>0 .
$$

We have

$$
\begin{gathered}
I_{w}\left(u_{k}\right)=\frac{1}{2}\left\|u_{k}\right\|_{\alpha}^{2}-\int_{0}^{T} F\left(t, u_{k}(t),{ }_{0} D_{t}^{\alpha} w(t)\right) d t, \\
I_{w}^{\prime}\left(u_{k}\right) u_{k}=\left\|u_{k}\right\|_{\alpha}^{2}-\int_{0}^{T} f\left(t, u_{k}(t),{ }_{0} D_{t}^{\alpha} w(t)\right) u_{k} d t .
\end{gathered}
$$


Then by (33) and $\left(H_{3}\right)$,

$$
\begin{aligned}
\left(\frac{1}{2}-\frac{1}{\mu}\right)\left\|u_{k}\right\|_{\alpha}^{2}= & I_{w}\left(u_{k}\right)-\frac{1}{\mu} I_{w}^{\prime}\left(u_{k}\right) u_{k} \\
& +\frac{\mu-1}{\mu} \int_{0}^{T} h(t) u_{k}(t) \cdot{ }_{0} D_{t}^{\alpha} w(t) d t \\
& +\int_{\left\{\left|u_{k}\right| \geq \xi_{0}\right\}} G\left(t, u_{k}(t),{ }_{0} D_{t}^{\alpha} w(t)\right) \\
& +\int_{\left\{\left|u_{k}\right| \leq \xi_{0}\right\}} G\left(t, u_{k}(t),{ }_{0} D_{t}^{\alpha} w(t)\right) \\
\leq & I_{w}\left(u_{k}\right)-\frac{u_{k}}{\mu} g\left(t, u_{k}(t),{ }_{0}^{\prime} D_{t}^{\alpha} w(t)\right) d t \\
& +\frac{C_{h} T^{\alpha}}{m \Gamma(\alpha+1)}\|w\|_{\alpha} \cdot\left\|u_{k}\right\|_{\alpha}+C_{3} \\
\leq & \bar{K}+\left[\frac{1}{\mu}\left\|I_{w}^{\prime}\left(u_{k}\right)\right\|+\frac{C_{h} T^{\alpha} K}{m \Gamma(\alpha+1)}\right]\left\|u_{k}\right\|_{\alpha}+C_{3}, \\
&
\end{aligned}
$$

where $C_{3}>0$. Combining with $I_{w}^{\prime}\left(u_{k}\right) \rightarrow 0$, as $k \rightarrow \infty$, we know that $\left\{u_{k}\right\}$ is bounded in $E_{0}^{\alpha}$.

Since $E_{0}^{\alpha}$ is a reflexive space, we can assume that $u_{k} \rightarrow u$. In $E_{0}^{\alpha}$, according to Remark 7 and Lemma 9 we have that $\left\{u_{k}\right\}$ is bounded in $C[0, T]$ and $\lim _{k \rightarrow \infty}\left\|u_{k}-u\right\|_{\infty}=0$. By the assumption $\left(\mathrm{H}_{2}\right)$, one gets

$$
\begin{aligned}
& \int_{0}^{T}\left[f\left(t, u_{k}(t),{ }_{0} D_{t}^{\alpha} w(t)\right)-f\left(t, u(t),{ }_{0} D_{t}^{\alpha} w(t)\right)\right] \\
& \quad \times\left(u_{k}-u\right) d t \\
& =\int_{0}^{T}\left[g\left(t, u_{k}(t),{ }_{0} D_{t}^{\alpha} w(t)\right)-g\left(t, u(t),{ }_{0} D_{t}^{\alpha} w(t)\right)\right] \\
& \quad \times\left(u_{k}-u\right) d t \\
& \longrightarrow 0, \quad k \longrightarrow \infty .
\end{aligned}
$$

Notice that

$$
\begin{aligned}
& {\left[I_{w}^{\prime}\left(u_{k}\right)-I_{w}^{\prime}(u)\right]\left(u_{k}-u\right)} \\
& \quad=I_{w}^{\prime}\left(u_{k}\right)\left(u_{k}-u\right)-I_{w}^{\prime}(u)\left(u_{k}-u\right) \\
& \quad \leq\left\|I_{w}^{\prime}\left(u_{k}\right)\right\| \cdot\left\|u_{k}-u\right\|_{\alpha}-I_{w}^{\prime}(u)\left(u_{k}-u\right) \\
& \quad \longrightarrow 0, \quad k \longrightarrow \infty .
\end{aligned}
$$

Moreover,

$$
\begin{aligned}
\left\|u_{k}-u\right\|_{\alpha}^{2}=\int_{0}^{T}[ & f\left(t, u_{k}(t),{ }_{0} D_{t}^{\alpha} w(t)\right) \\
& \left.\quad-f\left(t, u(t),{ }_{0} D_{t}^{\alpha} w(t)\right)\right]\left(u_{k}-u\right) d t \\
+ & {\left[I_{w}^{\prime}\left(u_{k}\right)-I_{w}^{\prime}(u)\right]\left(u_{k}-u\right), }
\end{aligned}
$$

so $\left\|u_{k}-u\right\|_{\alpha} \rightarrow 0$, as $k \rightarrow \infty$. That is, $\left\{u_{k}\right\}$ converges strongly to $u$ in $E_{0}^{\alpha}$.

Obviously, $I_{w}(0)=0$; therefore, by Mountain Pass theorem, $I_{w}$ has a nontrivial critical point $u_{w}$ in $E_{0}^{\alpha}$, with

$$
I_{w}\left(u_{w}\right)=\inf _{u \in \Gamma} \max _{t \in[0,1]} I_{w}(u(t)) \geq \alpha_{1}>0,
$$

where $\Gamma=\left\{u \in C\left([0,1], E_{0}^{\alpha}\right) \mid u(0)=0, u(1)=e\right\}$.

Step 2. We construct iterative sequence $\left\{u_{n}\right\}$ and estimate its norm in $E_{0}^{\alpha}$.

We consider the solutions $\left\{u_{n}\right\}$ of the following problem:

$$
\begin{gathered}
{ }_{t} D_{T}^{\alpha}\left(p(t){ }_{0} D_{t}^{\alpha} u_{n}(t)\right)=f\left(t, u_{n}(t),{ }_{0} D_{t}^{\alpha} u_{n-1}(t)\right), \\
t \in[0, T], \\
u_{n}(0)=u_{n}(T)=0,
\end{gathered}
$$

starting with $u_{1} \equiv 0$. By iterative technique, we can get a sequence of $\left\{u_{n}\right\}$, the nontrivial critical points obtained by Step 1, provided that there exists some constant $K>0$, such that $\left\|u_{n}\right\|_{\alpha} \leq K, n \geq 1$.

In the following, we estimate the norm of $\left\{u_{n}\right\}$ by the induction method. In fact, we need to prove that if we assume that for some $K>0,\left\|u_{n-1}\right\|_{\alpha} \leq K$, then $u_{n}$, the nontrivial critical point of $I_{u_{n-1}}$, also satisfies $\left\|u_{n}\right\|_{\alpha} \leq K$.

By the Mountain Pass characterization of the critical level, and $\left(H_{4}\right)$ and Cauchy's inequality with positive constant $\bar{\varepsilon}$, which is given in the sequel, it follows that

$$
\begin{aligned}
&\left|I_{u_{n-1}}\left(u_{n}\right)\right| \leq \max _{\tau \in[0, \infty)} I_{u_{n-1}}\left(\tau u^{*}\right) \\
& \leq \max _{\tau \in[0, \infty)}\left\{\frac{\tau^{2}}{2}-C_{1} \tau^{\mu} \int_{0}^{T}\left|u^{*}\right|^{\mu} d t\right. \\
&\left.+\tau C_{h} \int_{0}^{T}\left|{ }_{0} D_{t}^{\alpha} u_{n-1}\right| \cdot\left|u^{*}\right| d t+C_{2} T\right\} \\
& \leq \max _{\tau \in[0, \infty)}\left\{\frac{\tau^{2}}{2}-C_{1} \tau^{\mu}\left\|u^{*}\right\|_{L^{\mu}}^{\mu}\right. \\
&\left.+\tau \frac{C_{h} T^{\alpha}}{m \Gamma(\alpha+1)}\left\|u_{n-1}\right\|_{\alpha}+C_{2} T\right\} \\
& \leq \max _{\tau \in[0, \infty)}\left\{\left[\frac{1}{2}+\frac{\left[C_{h} T^{\alpha}\right]^{2}}{4 \bar{\varepsilon}[m \Gamma(\alpha+1)]^{2}}\right] \tau^{2}\right. \\
&\left.-C_{1} \tau^{\mu}\left\|u^{*}\right\|_{L^{\mu}}^{\mu}+C_{2} T\right\}+\bar{\varepsilon}\left\|u_{n-1}\right\|_{\alpha}^{2} .
\end{aligned}
$$


Let

$$
H(\tau):=\left[\frac{1}{2}+\frac{\left[C_{h} T^{\alpha}\right]^{2}}{4 \bar{\varepsilon}[m \Gamma(\alpha+1)]^{2}}\right] \tau^{2}-C_{1} \tau^{\mu}\left\|u^{*}\right\|_{L^{\mu}}^{\mu}+C_{2} T,
$$$$
\tau \geq 0 \text {, }
$$

and since $\mu>2, H(\tau)$ can achieve its maximum at some $\tau_{0}$. Hence

$$
\left|I_{u_{n-1}}\left(u_{n}\right)\right| \leq H\left(\tau_{0}\right)+\bar{\varepsilon} K^{2} .
$$

By (35), $\left\|u_{n-1}\right\|_{\alpha} \leq K$, and $I_{u_{n-1}}^{\prime}\left(u_{n}\right) u_{n}=0$, we have

$$
\begin{aligned}
\left(\frac{1}{2}-\frac{1}{\mu}\right)\left\|u_{n}\right\|_{\alpha}^{2} \leq & I_{u_{n-1}}\left(u_{n}\right)-\frac{1}{\mu} I_{u_{n-1}}^{\prime}\left(u_{n}\right) u_{n} \\
& +\frac{C_{h} T^{\alpha}}{m \Gamma(\alpha+1)}\left\|u_{n-1}\right\|_{\alpha} \cdot\left\|u_{n}\right\|_{\alpha}+C_{4} \\
\leq & H\left(\tau_{0}\right)+\bar{\varepsilon} K^{2}+\frac{C_{h} T^{\alpha} K}{m \Gamma(\alpha+1)}\left\|u_{n}\right\|_{\alpha}+C_{4},
\end{aligned}
$$

where $C_{4}>0$ is independent of $K$. For convenience, we denote $a=1 / 2-1 / \mu, b=C_{h} T^{\alpha} / m \Gamma(\alpha+1)$, and $c=H\left(\tau_{0}\right)+C_{4}$; then (44) can be written as

$$
a\left\|u_{n}\right\|_{\alpha}^{2}-b K\left\|u_{n}\right\|_{\alpha}-\left(c+\bar{\varepsilon} K^{2}\right) \leq 0,
$$

which implies

$$
\left\|u_{n}\right\|_{\alpha} \leq \frac{b K+\sqrt{b^{2} K^{2}+4 a\left(c+\bar{\varepsilon} K^{2}\right)}}{2 a} .
$$

Let

$$
\frac{b K+\sqrt{b^{2} K^{2}+4 a\left(c+\bar{\varepsilon} K^{2}\right)}}{2 a} \leq K,
$$

together with the assumption $a>b$; by simple calculation, for enough small $\bar{\varepsilon} \in(0, a-b)$ we obtain

$$
K \geq \sqrt{\frac{c}{a-b-\bar{\varepsilon}}} .
$$

Hence we can choose $K \in[\sqrt{c /(a-b-\bar{\varepsilon})}, \infty)$, such that $\left\|u_{n}\right\|_{\alpha} \leq K, n \geq 1$.

Step 3. We show that the iterative sequence $\left\{u_{n}\right\}$ constructed in Step 2 is convergent to a nontrivial solution of problem (3).

By Step 2 , we know $\left\|u_{n}\right\|_{\alpha} \leq K$, and by Remark 7, there exists positive constant $\rho_{1}$, such that

$$
\left\|u_{n}\right\|_{\infty} \leq \frac{T^{\alpha-1 / 2} K}{\Gamma(\alpha) \sqrt{m(2 \alpha-1)}}=: \rho_{1} .
$$

By (19), $I_{u_{n}}^{\prime}\left(u_{n+1}\right)\left(u_{n+1}-u_{n}\right)=0$, and $I_{u_{n-1}}^{\prime}\left(u_{n}\right)\left(u_{n+1}-u_{n}\right)=0$, we obtain

$$
\int_{0}^{T} p(t){ }_{0} D_{t}^{\alpha} u_{n+1} \cdot{ }_{0} D_{t}^{\alpha}\left(u_{n+1}-u_{n}\right) d t
$$

$$
\text { - } \int_{0}^{T} f\left(t, u_{n+1},{ }_{0} D_{t}^{\alpha} u_{n}\right)\left(u_{n+1}-u_{n}\right) d t=0,
$$

$$
\begin{aligned}
& \int_{0}^{T} p(t){ }_{0} D_{t}^{\alpha} u_{n} \cdot{ }_{0} D_{t}^{\alpha}\left(u_{n+1}-u_{n}\right) d t \\
& \quad-\int_{0}^{T} f\left(t, u_{n},{ }_{0} D_{t}^{\alpha} u_{n-1}\right)\left(u_{n+1}-u_{n}\right) d t=0 ;
\end{aligned}
$$

hence

$$
\begin{aligned}
& \int_{0}^{T} p(t)\left[{ }_{0} D_{t}^{\alpha}\left(u_{n+1}-u_{n}\right)\right]^{2} d t \\
& =\int_{0}^{T}\left[f\left(t, u_{n+1},{ }_{0} D_{t}^{\alpha} u_{n}\right)-f\left(t, u_{n},{ }_{0} D_{t}^{\alpha} u_{n-1}\right)\right]\left(u_{n+1}-u_{n}\right) d t .
\end{aligned}
$$

By $\left(H_{5}\right)$ it follows that

$$
\begin{aligned}
\left\|u_{n+1}-u_{n}\right\|_{\alpha}^{2} & \int_{0}^{T}\left[g\left(t, u_{n+1},{ }_{0} D_{t}^{\alpha} u_{n}\right)-g\left(t, u_{n},{ }_{0} D_{t}^{\alpha} u_{n-1}\right)\right]\left(u_{n+1}-u_{n}\right) d t \\
& +\int_{0}^{T} h(t){ }_{0} D_{t}^{\alpha}\left(u_{n}-u_{n-1}\right) \cdot\left(u_{n+1}-u_{n}\right) d t \\
\leq & L_{1} \int_{0}^{T}\left|u_{n+1}-u_{n}\right|^{2} d t \\
& +\left(L_{2}+C_{h}\right) \int_{0}^{T}\left|{ }_{0} D_{t}^{\alpha}\left(u_{n}-u_{n-1}\right)\right| \cdot\left|u_{n+1}-u_{n}\right| d t \\
\leq & \frac{L_{1} T^{2 \alpha}}{m[\Gamma(\alpha+1)]^{2}}\left\|u_{n+1}-u_{n}\right\|_{\alpha}^{2} \\
& +\left(L_{2}+C_{h}\right)\left\|{ }_{0} D_{t}^{\alpha}\left(u_{n}-u_{n-1}\right)\right\|_{L^{2}} \cdot\left\|u_{n+1}-u_{n}\right\|_{L^{2}} \\
\leq & \frac{L_{1} T^{2 \alpha}}{m[\Gamma(\alpha+1)]^{2}}\left\|u_{n+1}-u_{n}\right\|_{\alpha}^{2} \\
& +\frac{\left(L_{2}+C_{h}\right) T^{\alpha}}{m \Gamma(\alpha+1)}\left\|u_{n}-u_{n-1}\right\|_{\alpha} \cdot\left\|u_{n+1}-u_{n}\right\|_{\alpha} ;
\end{aligned}
$$

hence

$$
\begin{gathered}
\left(1-\frac{L_{1} T^{2 \alpha}}{m[\Gamma(\alpha+1)]^{2}}\right)\left\|u_{n+1}-u_{n}\right\|_{\alpha} \\
\leq \frac{\left(L_{2}+C_{h}\right) T^{\alpha}}{m \Gamma(\alpha+1)}\left\|u_{n}-u_{n-1}\right\|_{\alpha} .
\end{gathered}
$$

Since $0<L<1$, we know that $\left\{u_{n}\right\}$ is a Cauchy sequence in $E_{0}^{\alpha}$, so there exists a $u \in E_{0}^{\alpha}$ such that $\left\{u_{n}\right\}$ converges strongly to $u$ in $E_{0}^{\alpha}$. 
In order to show that $u$ is a solution of problem (3), we need to prove that

$$
\begin{array}{rl}
\int_{0}^{T} & p(t){ }_{0} D_{t}^{\alpha} u(t){ }_{0} D_{t}^{\alpha} v(t) d t \\
\quad=\int_{0}^{T} f\left(t, u(t),{ }_{0} D_{t}^{\alpha} u(t)\right) v(t) d t, \quad \forall v(t) \in E_{0}^{\alpha} .
\end{array}
$$

It suffices to show that

$$
\int_{0}^{T} f\left(t, u_{n},{ }_{0} D_{t}^{\alpha} u_{n-1}\right) v(t) d t \longrightarrow \int_{0}^{T} f\left(t, u,{ }_{0} D_{t}^{\alpha} u\right) v(t) d t
$$

Indeed, it follows from the assumption $\left(\mathrm{H}_{5}\right)$ that

$$
\begin{aligned}
\int_{0}^{T}\left[f\left(t, u_{n},{ }_{0} D_{t}^{\alpha} u_{n-1}\right)-f\left(t, u,{ }_{0} D_{t}^{\alpha} u\right)\right] v(t) d t \\
=\int_{0}^{T}\left[g\left(t, u_{n},{ }_{0} D_{t}^{\alpha} u_{n-1}\right)-g\left(t, u,{ }_{0} D_{t}^{\alpha} u\right)\right] v(t) d t \\
\quad+\int_{0}^{T} h(t)_{0} D_{t}^{\alpha}\left(u_{n-1}-u\right) v(t) d t \\
\leq L_{1} \int_{0}^{T}\left|u_{n}-u\right| \cdot|v(t)| d t \\
\quad+\left(L_{2}+C_{h}\right) \int_{0}^{T}\left|{ }_{0} D_{t}^{\alpha}\left(u_{n-1}-u\right)\right| \cdot|v(t)| d t \\
\leq \\
\quad\left[\frac{L_{1} T^{2 \alpha}}{m[\Gamma(\alpha+1)]^{2}}\left\|u_{n}-u\right\|_{\alpha}\right. \\
\left.\quad+\frac{\left(L_{2}+C_{h}\right) T^{\alpha}}{m \Gamma(\alpha+1)}\left\|u_{n-1}-u\right\|_{\alpha}\right] \cdot\|v\|_{\alpha} \\
\longrightarrow 0, \quad n \longrightarrow \infty .
\end{aligned}
$$

Then, $u(t)$ is a solution of $(3)$, and since $I_{u_{n-1}}\left(u_{n}\right) \geq \alpha_{1}>0$ for $n=2,3, \ldots$, we obtain that $u(t)$ is a nontrivial solution of problem (3).

\section{Example}

In this section, we provide an explicit example to illustrate our main results.

Example 1. Consider the following fractional boundary value problem:

$$
\begin{gathered}
{ }_{t} D_{1}^{0.75}\left({ }_{0} D_{t}^{0.75} u(t)\right)= \\
t \in[0,1], \\
u(0)=u(1)=0 .
\end{gathered}
$$

Obviously, $\alpha=0.75, f(t, \xi, \zeta)=\xi^{3}(2+\sin \zeta), p(t)=1$, and $h(t)=0$. Let $C_{0}=r=3, \mu=4$, and $C_{1}=1 / 4$, for all $\xi_{0}$,
$\mathrm{C}_{2}>0$; it is not difficult to verify that function $f$ satisfies the assumptions $\left(H_{1}\right)-\left(H_{4}\right)$.

For $\left(H_{5}\right)$, for all $t \in[0, T], \xi_{1}, \xi_{2} \in\left[-\rho_{1}, \rho_{1}\right], \zeta_{1}, \zeta_{2} \in R$, it follows that

$$
\begin{aligned}
\mid f(t, & \left.\xi_{1}, \zeta_{1}\right)-f\left(t, \xi_{2}, \zeta_{2}\right) \mid \\
\leq & \left|\xi_{1}^{3}\left(2+\sin \zeta_{1}\right)-\xi_{1}^{3}\left(2+\sin \zeta_{2}\right)\right| \\
& \quad+\left|\xi_{1}^{3}\left(2+\sin \zeta_{2}\right)-\xi_{2}^{3}\left(2+\sin \zeta_{2}\right)\right| \\
\leq & 9 \rho_{1}^{2}\left|\xi_{1}-\xi_{2}\right|+\rho_{1}^{3}\left|\zeta_{1}-\zeta_{2}\right| .
\end{aligned}
$$

Then we choose $L_{1}=9 \rho_{1}^{2}, L_{2}=\rho_{1}^{3}$, where $\rho_{1}=\sqrt{2} K / \Gamma(0.75)$. Since $h(t)=0$, it obviously follows that $1 / 2-1 / \mu>0$ and that (25) is valid. It suffices to verify that

$$
L=\frac{\Gamma(1.75) L_{2}}{[\Gamma(1.75)]^{2}-L_{1}}=\frac{1.5 \sqrt{2} K^{3}}{(0.75)^{2}[\Gamma(0.75)]^{4}-18 K^{2}} \in(0,1) .
$$

From (48), now we estimate the value of $c=H\left(\tau_{0}\right)+C_{4}$, where $\mathrm{C}_{4}$ is dependent on $\xi_{0}$ and

$$
H(\tau)=\frac{1}{2} \tau^{2}-\frac{1}{4} \tau^{4} \int_{0}^{1}\left|u^{*}(t)\right|^{4} d t+C_{2}, \quad \tau \geq 0 .
$$

Since $\int_{0}^{1}\left|{ }_{0} D_{t}^{0.75}\left(t^{2}-t\right)\right|^{2} d t=\left(1 /[\Gamma(1.25)]^{2}\right)(2 / 3-96 / 175)$, we can choose $u^{*}(t)=(\Gamma(1.25) / \sqrt{2 / 3-96 / 175})\left(t^{2}-t\right) \approx$ 2.6375745( $\left.t^{2}-t\right)$, such that $u^{*}(t) \in E_{0}^{0.75}$ with $\left\|u^{*}(t)\right\|_{0.75}=1$.

By direct calculation, we have that $\tau_{0}=$ $1 / \sqrt{\int_{0}^{1}\left|u^{*}(t)\right|^{4} d t} \approx 0.1853281 ;$ moreover,

$$
H\left(\tau_{0}\right)=\frac{1}{4} \tau_{0}^{2}+C_{2} \approx 0.008586625+C_{2} .
$$

Then $c \approx 0.008586625+C_{2}+C_{4}$; by the arbitrariness of $\xi_{0}$ and $C_{2}$, we can choose enough small $\xi_{0}, C_{2}>0$, such that $c=0.01$, $a=1 / 4, b=0$, and $\bar{\varepsilon}=0.1$.

Let $K=\sqrt{c /(a-b-\bar{\varepsilon})}=1 / \sqrt{15} \approx 0.2581989$; we get

$$
L=\frac{0.03651484}{1.2684044-1.2000001} \approx 0.5338091 \in(0,1) \text {. }
$$

Then all conditions of Theorem 12 are satisfied. Consequently we obtain that problem (57) has a nontrivial solution.

\section{Conflict of Interests}

The authors declare that there is no conflict of interests regarding the publication of this paper.

\section{Authors' Contribution}

Wenzhe Xie and Jing Xiao contributed equally to this work. 


\section{Acknowledgments}

This work is supported by the Scientific Research Fund of Hunan Provincial Education Department (13K029), the Innovation Fund Project For Graduate Student of Hunan Province (CX2013B219), partially supported by the National Natural Science Foundation of China (61170320), and Nature Science Foundation of Guangdong Medical College (B2012053).

\section{References}

[1] V. Lakshmikantham and A. S. Vatsala, "Basic theory of fractional differential equations," Nonlinear Analysis: Theory, Methods \& Applications A: Theory and Methods, vol. 69, no. 8, pp. 2677-2682, 2008.

[2] V. Lakshmikantham and A. S. Vatsala, "General uniqueness and monotone iterative technique for fractional differential equations," Applied Mathematics Letters, vol. 21, no. 8, pp. 828-834, 2008.

[3] A. A. Kilbas, H. M. Srivastava, and J. J. Trujillo, Theory and Applications of Fractional Differential Equations, Elsevier Science, Amsterdam, The Netherlands, 2006.

[4] B. Ahmad, J. J. Nieto, A. Alsaedi, and M. El-Shahed, "A study of nonlinear Langevin equation involving two fractional orders in different intervals," Nonlinear Analysis: Real World Applications, vol. 13, no. 2, pp. 599-606, 2012.

[5] J. S. Leszczynski and T. Blaszczyk, "Modeling the transition between stable and unstable operation while emptying a silo," Granular Matter, vol. 13, no. 4, pp. 429-438, 2011.

[6] E. Szymanek, "The application of fractional order differential calculus for the description of temperature profiles in a granular layer," in Advances in the Theory and Applications of Non-integer Order Systems, vol. 257 of Lecture Notes in Electrical Engineering, pp. 243-248, 2013.

[7] F. Jiao and Y. Zhou, "Existence results for fractional boundary value problem via critical point theory," International Journal of Bifurcation and Chaos in Applied Sciences and Engineering, vol. 22, no. 4, Article ID 1250086, 2012.

[8] H. R. Sun and Q. G. Zhang, "Existence of solutions for a fractional boundary value problem via the Mountain Pass method and an iterative technique," Computers \& Mathematics with Applications, vol. 64, no. 10, pp. 3436-3443, 2012.

[9] F. Jiao and Y. Zhou, "Existence of solutions for a class of fractional boundary value problems via critical point theory," Computers \& Mathematics with Applications, vol. 62, no. 3, pp. 11811199, 2011.

[10] C. Bai, "Infinitely many solutions for a perturbed nonlinear fractional boundary-value problem," Electronic Journal of Differential Equations, vol. 2013, pp. 1-12, 2013.

[11] J. Chen and X. H. Tang, "Existence and multiplicity of solutions for some fractional boundary value problem via critical point theory," Abstract and Applied Analysis, vol. 2012, Article ID 648635, 21 pages, 2012.

[12] C. Torres, "Mountain pass solution for a fractional boundary value problem," Journal of Fractional Calculus and Applications, vol. 5, no. 1, pp. 1-10, 2014.

[13] D. de Figueiredo, M. Girardi, and M. Matzeu, "Semilinear elliptic equations with dependence on the gradient via mountainpass techniques," Differential and Integral Equations, vol. 17, no. 1-2, pp. 119-126, 2004.
[14] K. Teng and C. Zhang, "Existence of solution to boundary value problem for impulsive differential equations," Nonlinear Analysis: Real World Applications, vol. 11, no. 5, pp. 4431-4441, 2010. 


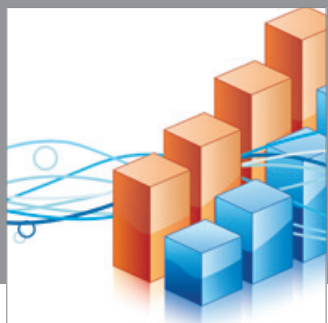

Advances in

Operations Research

mansans

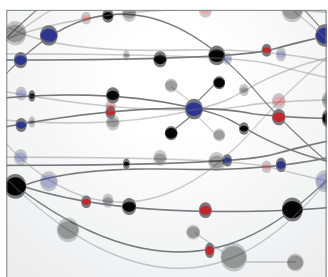

The Scientific World Journal
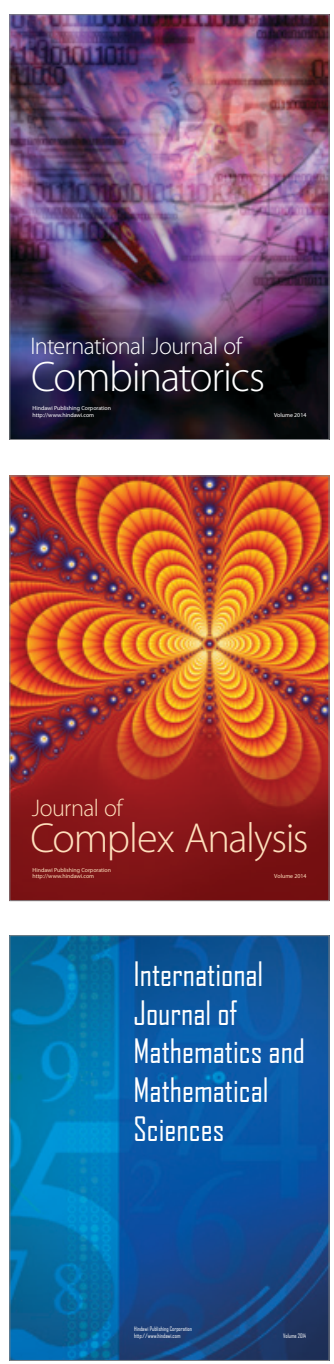
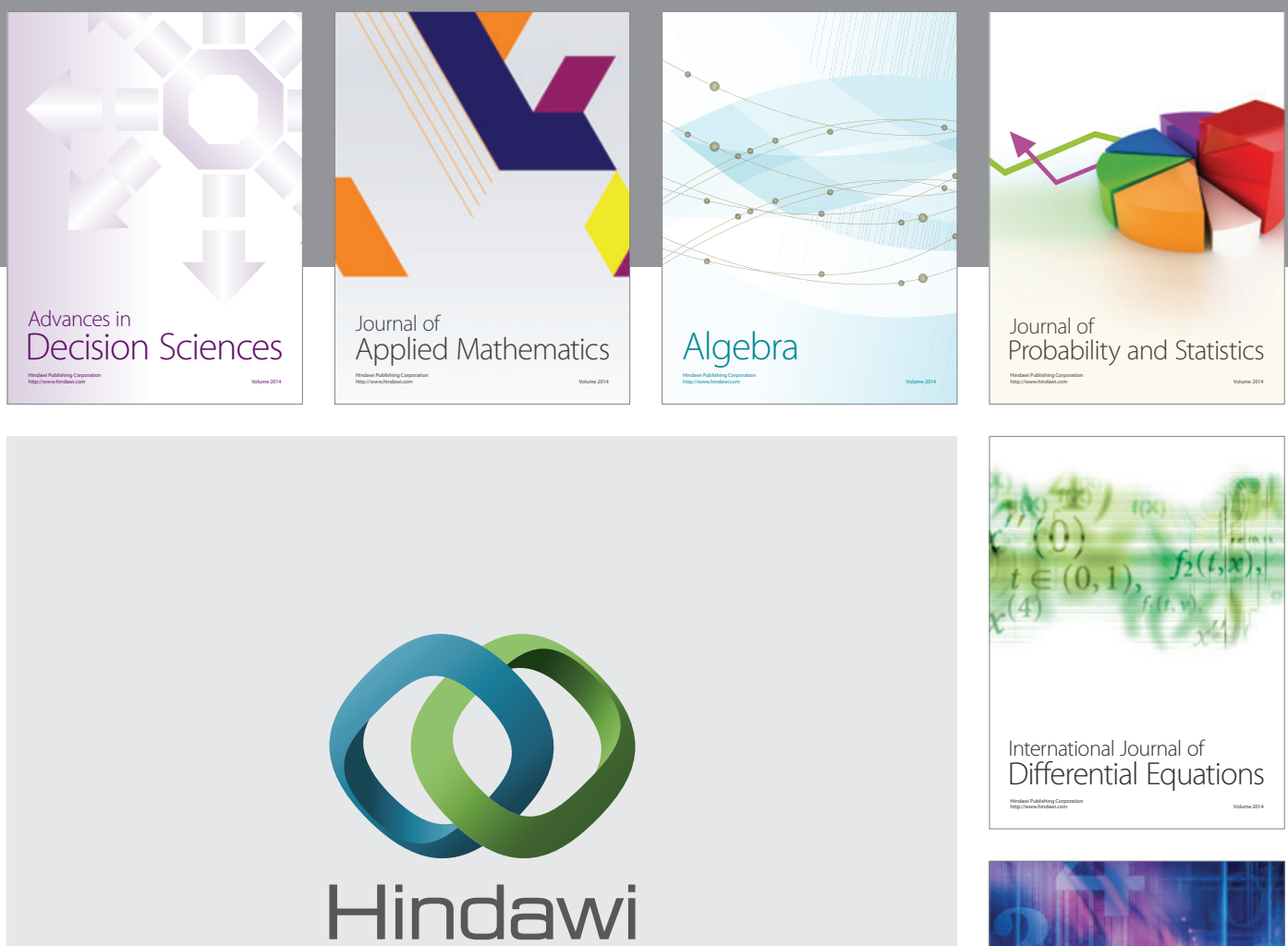

Submit your manuscripts at http://www.hindawi.com
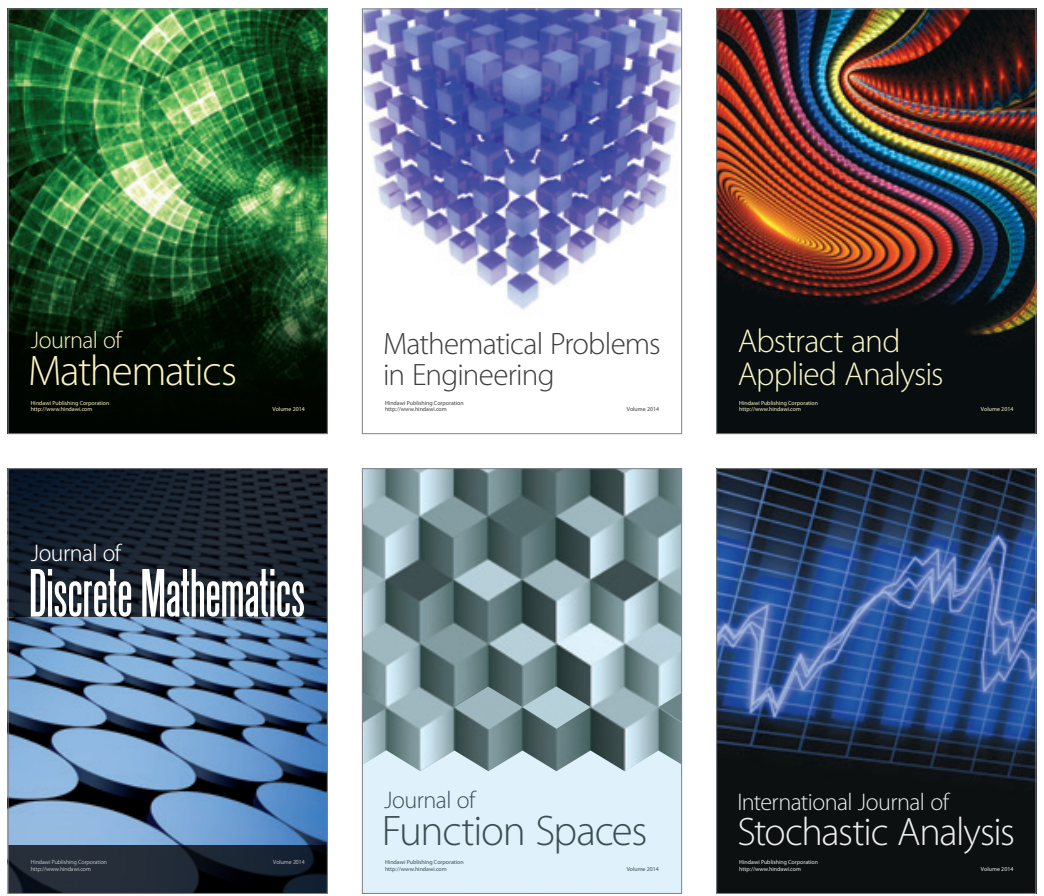

Journal of

Function Spaces

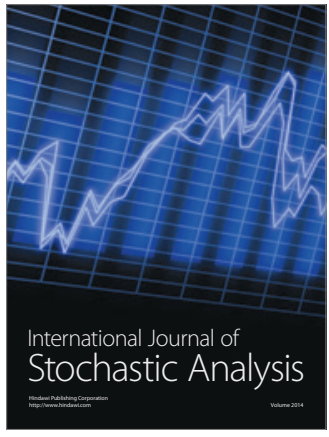

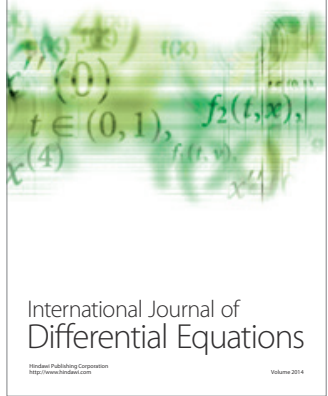
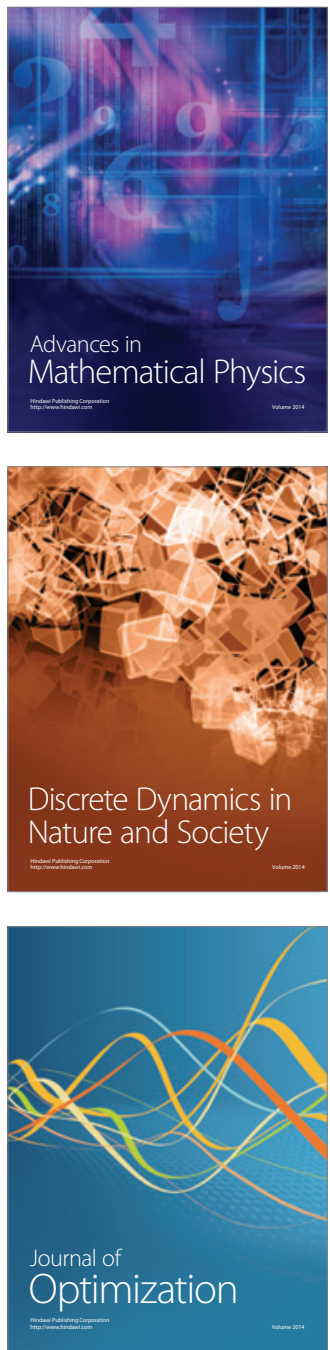